Daša Stanič

Filozofska fakulteta, Univerza v Ljubljani

dasa.stanic@ff.uni-lj.si
UDK 811.131.1'271.1:378(497.4)

DOI: 10.4312/vestnik.9.255-285

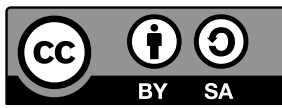

\title{
ANALISI DEGLI ERRORI NELLA PRODUZIONE SCRITTA DEGLI STUDENTI DI ITALIANO COME LS A LIVELLO UNIVERSITARIO
}

INTRODUZIONE

Il presente contributo si propone di analizzare gli errori nella produzione scritta degli studenti del secondo anno del corso di Laurea Triennale in Lingua e letteratura italiana ${ }^{1}$ della Facoltà di Lettere e Filosofia di Lubiana con lo scopo di individuare e descrivere gli errori più frequenti e di stabilire, qualora possibile, le loro cause. In una seconda fase, i risultati dell'analisi verranno usati per progettare e valutare l'insegnamento e i corsi Italijanske lektorske vaje 1 e Italijanske lektorske vaje 2.

Per chi decide di studiare l'italiano alla facoltà e diventare quindi esperto nel campo, l'obiettivo nei primi tre anni di studio è quello di raggiungere almeno il livello B2 stabilito dal Quadro comune europeo di riferimento per le lingue (QCER 2002) ossia avvicinarsi il più possibile ai due livelli più alti (C1 e $\mathrm{C} 2)$ di competenza linguistica. Inoltre, è imprescindibile, specialmente in un contesto di studio universitario, che gli studenti sviluppino, oltre a una buona competenza linguistica e comunicativa, anche una buona competenza metalinguistica. Per tutti questi motivi sono inclusi nel piano di studi del corso di Lingua e letteratura italiana, nei primi due anni, oltre ai corsi Italijanske lektorske vaje 1 e $2^{2}$ (in italiano Esercitazioni di lingua italiana 1 e 2), corsi approfonditi di morfologia e sintassi italiana e di analisi contrastiva, durante i quali gli studenti studiano in dettaglio le regole morfosintattiche ${ }^{3}$ dell'italiano standard e le differenze tra

1 Il corso di Lingua e letteratura italiana è un corso a doppio titolo. Molti dei nostri studenti studiano, oltre all'italiano, un'altra lingua straniera. Le combinazioni più frequenti sono con lo spagnolo, l'inglese, il francese, il tedesco, ma anche con il cinese e il giapponese. Inoltre, molti studenti frequentano lettorati di varie lingue straniere come corsi a scelta.

2 Italijanske lektorske vaje1, 2 e 3 sono corsi il cui fine è praticare le quattro abilità linguistiche (leggere, ascoltare, parlare e scrivere) e conoscere alcuni aspetti culturali dell'Italia.

3 Durante le lezioni, che si basano su alcune delle grammatiche italiane (Grammatica italiana di Dardano e Trifone (2003), Grammatica italiana di Serianni (2010), ecc.), le regole dell'italiano vengono esposte e spiegate agli studenti in modo lineare e deduttivo, cioè vengono presentate le regole, le sottoregole, le eccezioni e viene illustrato l'uso. La spiegazione è seguita da esercizi, spesso di tipo ricettivo o ricettivo-produttivo (cf. Mertelj, 2003) il cui scopo è consolidare le conoscenze delle forme grammaticali e il loro significato. Generalmente non ne viene richiesto un libero uso in contesti comunicativi. 
l'italiano e lo sloveno (lingua madre o L1 per la maggioranza degli studenti). I corsi sono seguiti da esami in cui si valuta l'applicazione pratica e la conoscenza teorica. Non raramente è possibile notare che, anche dopo aver frequentato tutti questi corsi, gli studenti fanno ancora molti errori nella produzione scritta e orale, spesso non raggiungendo gli obiettivi prestabiliti.

A nostro parere un insegnamento che si proponga di essere efficace non può e non deve in alcun modo trascurare gli errori degli apprendenti, poiché per il raggiungimento di un livello superiore di competenza linguistica, nel contesto dell'apprendimento di una LS in ambito universitario, sono indispensabili la consapevolezza dei propri errori e la riflessione linguistica su di essi da parte dell'apprendente, guidata da un insegnante che conosca gli errori tipici dei propri studenti.

Che gli apprendenti commettano, nel processo di apprendimento di una LS, sia errori nella comprensione che nella produzione è inevitabile visto che l'apprendimento di una lingua straniera è un processo graduale e individuale. Non è, quindi, possibile aspettarsi che le strutture "appaiano" nell'interlingua dell'apprendente da subito, una dopo l'altra, come pienamente sviluppate e senza errori (Larsen-Freeman 2001: 255). Gli errori nella comprensione sono stati studiati meno che quelli nella produzione proprio perché sarebbe molto più difficile scoprirne le cause (cf. Ellis 1994: 86). È comunque vero che anche nella produzione, sia orale che scritta, non è facile né scoprire le cause di un errore né individuare l'errore in un primo momento. È noto che spesso perfino i parlanti madrelingua hanno problemi a stabilire se una forma sia sbagliata oppure no (Lennon, 1991: 181-182, Cattana e Nesci 2004: 36).

\subsection{L'errore nei diversi metodi di insegnamento della LS}

Russell (2009) nel suo articolo menziona la lunga e controversa storia della concezione e della correzione degli errori nel campo della didattica delle LS. Spesso il modo di concepire e trattare gli errori dipende soprattutto dai concetti, principi e metodi sui quali l'insegnante basa il proprio insegnamento. I metodi dell'insegnamento/apprendimento di una LS che nella storia si sono susseguiti sono molto diversi tra di loro, tanto che alcuni si prefiggono addirittura degli obiettivi diametralmente opposti. Di conseguenza differiscono anche le varie concezioni degli errori e della loro correzione. Per lungo tempo l'errore è stato considerato nella glottodidattica delle LS il "prodotto" di un apprendimento imperfetto o inappropriato, una devianza dalla norma e sicuramente un elemento da correggere e da eradicare nel processo di apprendimento. Lo scopo principale del 
metodo grammaticale-traduttivo era appunto la correttezza grammaticale a scapito di altri obiettivi possibili nell'apprendimento di una LS. Negli anni Settanta del XX secolo l'atteggiamento verso l'errore cambia come diretta conseguenza di un modo diverso di concepire l'insegnamento/apprendimento di una LS. L'errore assume così per la prima volta una connotazione positiva e diventa, da elemento da evitare, un elemento da sfruttare per l'apprendimento/insegnamento di una LS.

Nell'analisi contrastiva il confronto della LS con la L1 ci permette di indentificare e prevedere - e pertanto di evitare - le difficoltà (e quindi anche gli errori) che un apprendente incontra durante il processo di apprendimento. Secondo i contrastivisti vale il seguente principio: più la L1 e la LS sono diverse, più errori si verificheranno nell'apprendimento della LS, poiché è proprio la L1 la fonte di tutti gli errori che l'apprendente commette nella LS (cf. Gass e Selinker 2008: 96). L'errore viene considerato un elemento decisamente negativo che va prevenuto e, quando individuato, deve essere subito corretto. L'atteggiamento contrastivista ha un peso importante nella glottodidattica, ma si è rivelato non privo di limitazioni. Cattana e Nesci (2004: 29), infatti, affermano che l'analisi contrastiva dimostra un certa validità a livello fonetico, ma non a livello morfosintattico e morfologico. Inoltre, essa è in grado di prevedere solo alcuni errori degli apprendenti mentre non riesce a stabilire le cause per molte altre tipologie di errori (D'Annunzio e Serragiotto 2007).

La concezione dell'errore nell'apprendimento/insegnamento di una LS è cambiata radicalmente anche grazie all'articolo The significance of learners' errors di Corder, pubblicato nel 1967. In quel periodo non cambia solo il modo di concepire l'errore ma anche il modo di vedere l'insegnamento/apprendimento delle LS: si passa dal comportamentismo, in cui l'apprendente deve acquisire corrette abitudini linguistiche, al cognitivismo, che mette al centro del processo di apprendimento un apprendente attivo che secondo Corder (1967: 166) nel processo di apprendimento di una LS costruisce in varie fasi di apprendimento vari sistemi linguistici transitori (chiamati di seguito da Corder dialetti idiosincratici, 1981: 14) servendosi delle stesse strategie di apprendimento che usa durante l'apprendimento della L1. Si tratta di sistemi non ancora perfetti ${ }^{4}$, ma definiti, sistematici, regolari e dunque con una logica interna. Selinker (1972) introduce il termine interlingua con cui si riferisce ad un concetto quasi analogo al sistema linguistico transitorio di Corder. L'apprendente, un partecipante attivo nel processo di apprendimento, fa delle generalizzazioni sulla lingua che sta imparando e costruisce, in un determinato momento dell'apprendimento, un sistema linguistico provvisorio a seguito delle ipotesi che fa sulla lingua. Anche l'errore, in un sistema del genere, deve necessariamente essere sistematico e regolare, dato che si manifesta quando lo studente cerca di imporre le proprie regolarità alla lingua a cui viene esposto in base ai mezzi

4 L'apprendente non ha ancora a disposizione tutti i mezzi di una LS ma solo quelli che ha studiato e interiorizzato fino a quel momento. 
che conosce (Gass e Selinker 2008, 102). L'apprendente non è in grado di identificare e (auto)correggere gli errori sistematici (dovuti a una mancata competenza) dato che li percepisce, in relazione alla sua competenza transitoria, come forme corrette e non come errori. L'errore diventa così l'indice del sistema transitorio, ed è uno degli elementi che ci permettono di ricostruire la competenza linguistica di un apprendente in un dato momento dell'apprendimento. Analizzando gli errori il valutatore può stabilire quanto l'apprendente si sia avvicinato all'obiettivo, cioè al livello della competenza linguistica da raggiungere, e che cosa deve ancora studiare. Per lo studente, invece, l'errore rappresenta l'opportunità per imparare, siccome gli permette di sperimentare e valutare le proprie ipotesi sulla lingua. Nel metodo comunicativo, il cui obiettivo principale è l'acquisizione della competenza comunicativa, la lingua viene vista principalmente come strumento di comunicazione e di azione sociale. Il suo aspetto pragmatico (capire le intenzioni, usare la lingua adeguata alle situazioni e agli scopi) prevale su quello formale, ovvero i due aspetti diventano equilibrati quanto mai prima. Gli errori non sono al centro dell'attenzione, e quando vengono analizzati ciò avviene considerando il contesto comunicativo. Possono, comunque, rappresentare un valido aiuto e sostegno nell'apprendimento/insegnamento di una LS ed è auspicabile che gli apprendenti sviluppino degli strumenti per diventare consci dei propri errori ed essere capaci a validare la propria competenza linguistica anche in base agli errori che commettono (Cattana e Nesci 2004: 30). Esistono anche approcci in cui la correzione degli errori viene vista come dannosa. Si tratta degli approcci umanistico-affettivi presentati e denominati da Pawlak (2014: 9) come zero grammar option, che iniziano ad affermarsi negli anni Settanta e Ottanta del XX secolo. Questi approcci mettono al centro dell'apprendimento di una LS l'aspetto affettivo, visto che è proprio il fattore affettivo quello che può sia agevolare notevolmente sia compromettere in modo decisivo il processo di apprendimento. Krashen (1982: 74) è del parere che gli errori siano inevitabili, e che la correzione degli errori come "tipica reazione dell'insegnante" sia un serio sbaglio e molto dannosa in quanto influisce negativamente sul filtro affettivo dell'apprendente. Secondo Krashen (riportato anche in Russell 2009: 22) avranno molto più successo nell'apprendimento gli studenti altamente motivati, sicuri di sé e meno ansiosi. Anche nel QCER (2002: 189 e 199) viene dedicato agli errori un capitolo in cui vengono definiti i tipi di errori (cf. par. 2.2) e di seguito vengono esposti alcuni modi in cui è possibile agire di fronte ad essi e le loro possibili interpretazioni. Secondo il QCER (2002: 199) l'osservazione e l'analisi degli errori possono essere utili per progettare l'insegnamento e i corsi, per elaborare materiali e per valutare l'insegnamento e l'apprendimento di una LS.

A questo punto va menzionato un altro fenomeno constatato negli ultimi decenni, in particolare nell'ambito delle strategie di apprendimento: gli studenti, qualora sottoposti alla correzione, cercheranno di evitare gli errori anche usando costrutti per loro "facili" (più semplici dal punto di vista morfosintattico e stilistico) anche se tali costrutti, in effetti, non rispecchiano la loro reale competenza linguistica generale. 


\subsection{La definizione e l'identificazione dell'errore}

Nell'ambito della glottodidattica è molto diffusa la definizione dell'errore di Lennon (1991: 182) che mette al centro il parlante madrelingua come quello che decide se una forma o una combinazione di forme sia corretta oppure no e definisce l'errore come:

"...a linguistic form or combination of forms which, in the same context and under similar conditions of production, would, in all likelihood, not be produced by the speakers' native speakers counterparts."

Molte definizioni dell'errore includono come criterio proprio il confronto con il parlante madrelingua, benché anche la "norma del parlante madrelingua" abbia le proprie debolezze. Spesso, infatti, esistono diverse varianti di una lingua, i dialetti e molti altri fattori che influenzano il modo di parlare di ogni singola persona in contesti diversi (cf. Pawlak 2014: 4-5). Serianni (2006: 33) ne menziona alcuni:

"Tra i due poli "giusto"/"sbagliato" si situa una zona grigia, in cui il parlante nativo può avere dubbi e incertezze, dipendenti da vari fattori: la sua cultura e il conseguente grado di sicurezza linguistica che ne scaturisce; la sensibilità per fatti di lingua e l'aspirazione al prestigio sociolinguistico; il contesto in cui agisce (le preoccupazioni normative saranno minime nell'ambiente familiare o nei "gruppi di pari", massime in condizioni formali, per esempio interagendo con un esaminatore o con un superiore gerarchico)."

L'errore dal punto di vista dell'interlingua è una delle componenti imprescindibili dell'apprendimento di una LS ed è infatti la prova dell'interlingua (sistema provvisorio non ancora perfetto), come lo è anche la fossilizzazione (Selinker 1972: 215).

Quando parliamo di errori è necessario fare una distinzione tra i diversi tipi di errori, o meglio la distinzione tra l'errore e lo sbaglio. Se l'errore, dovuto all'interlingua, è sistematico e l'apprendente non è in grado di correggerlo, lo sbaglio, al contrario, è momentaneo, non sistematico e l'apprendente è capace di correggerlo da solo. È possibile trovare una simile distinzione anche nel QCER (2002: 189-190) in cui gli tutti gli errori che gli apprendenti fanno vengono divisi in errori e sbagli. I primi, dovuti all'interlingua, sono definiti come errori di apprendimento e i secondi, quelli che si verificano quando l'apprendente non riesce ad attivare le proprie competenze nel modo dovuto, come errori di produzione. L'errore (e non lo sbaglio), qualora non venga corretto attraverso la correzione esterna nel processo di apprendimento, può fossilizzarsi (cf. Long 2003:513; Mezzadri 2015: 271) e stabilizzarsi nella lingua dell'apprendente indipendentemente dalla quantità di lingua a cui viene esposto (cf. Selinker 1972: 229). La non correzione degli errori è comunque solo uno dei molti fattori che portano alla fossilizzazione (Long 2003: 513). 
Quindi, per identificare l'errore si deve tenere conto di diversi fattori; fatto che rende spesso il processo faticoso. Di conseguenza, è logico che sia i parlanti madrelingua che i professori di lingua mostrino spesso variabilità nell'identificazione degli errori. Nel contesto dell'insegnamento/apprendimento di una LS emergono, oltre ai fattori già menzionati sopra, altri elementi che vanno considerati: la concezione dell'errore da parte dell'insegnante, l'atteggiamento dell'insegnante verso gli errori, la sua competenza linguistica, lo scopo dell'attività che devono svolgere gli studenti, ecc. Inoltre, è più difficile identificare gli errori nella lingua parlata, e la difficoltà aumenta con l'aumentare del grado di informalità (Lennon, 1991).

Cattana e Nesci (2004: 38) offrono cinque criteri in base ai quali è possibile identificare un errore:

a) Il criterio della correttezza, secondo il quale ogni deviazione dal sistema di regole deve essere considerata inaccettabile e quindi un errore. Il criterio della correttezza è strettamente legato alla definizione della norma e al rapporto tra la norma e l'uso, rapporto molto complesso in italiano a causa dell'esistenza di molte varianti e di molti dialetti.

b) Il criterio dell'appropriatezza è quello che valuta la capacità dell'apprendente di usare la varietà di lingua adeguata alle circostanze, agli interlocutori o all'intenzione comunicativa. Un parlante non madrelingua, qualora voglia diventare parte attiva in una comunità diversa dalla sua, deve necessariamente sviluppare, oltre alla competenza grammaticale, quella comunicativa, cioè quella composta da più tipi di competenze: linguistiche, socioculturali e pragmatiche (QCER 2002: 133-134)5.

c) Il criterio della comprensibilità definisce come errore tutto quello che ostacola $\mathrm{o}$ rende impossibile la comunicazione. Un enunciato, anche nel caso in cui contenga più errori grammaticali o lessicali, può essere comunque comprensibile e quindi definito come "corretto" secondo questo criterio. Si distinguono, in base al criterio della comprensibilità, gli errori globali e gli errori locali. I primi influiscono sull'organizzazione globale del testo e rendono il messaggio difficilmente comprensibile o incomprensibile, i secondi invece non ostacolano la comprensione. Vengono classificati in questo secondo gruppo alcuni errori nella flessione, l'omissione di elementi non portatori di significato, ecc.

d) Il criterio della soggettività nel valutare l'errore. È noto che ogni insegnante giudica una forma o un enunciato come accettabile o inaccettabile (corretto o sbagliato) in base ad una "norma soggettiva".

e) Il criterio della flessibilità permette all'insegnante di decidere in base a vari fattori (il contesto comunicativo, i bisogni linguistici degli apprendenti, il livello di apprendimento, la personalità dell'apprendente, il tipo di attività, ecc.) se tollerare un errore oppure correggerlo.

5 La situazione linguistica italiana, tanto complessa, influenza anche il criterio dell'appropriatezza. La lingua, dunque, non va adattata solo a diverse situazioni comunicative ma anche alla regione geografica, alla condizione sociale dei parlanti e al mezzo di comunicazione usato (Cattana e Nesci 2004: 46 e 47). 
Per identificare e valutare gli errori spesso è necessario combinare i criteri sopra descritti (eccetto in un'analisi focalizzata su uno degli aspetti). La correttezza grammaticale non serve a nulla se lo studente non sviluppa anche la competenza comunicativa. Da solo, il criterio della comprensibilità non basta se alcuni errori che si ripetono (pur non ostacolando la comprensibilità) si fossilizzano e si fissano nella lingua dell'apprendente e, a lungo termine, possono compromettere in modo significativo il suo passaggio verso un livello più alto di competenza linguistica. In un'attività comunicativa orale, probabilmente, si darà, più importanza all'appropriatezza e alla comprensibilità che alla correttezza; quest'ultima sarà invece più importante in un compito scritto.

\subsection{Le cause degli errori}

Parlando delle cause degli errori Taylor (1986: 159) sottolinea l'importanza di un approccio che vada oltre le cause psicologiche. Secondo lui le cause si dividono in quattro gruppi: cause psicolinguistiche (la natura della LS e i problemi degli apprendenti nella produzione della LS), cause sociolinguistiche (l'incapacità di adattare la lingua al contesto sociale), cause epistemiche (mancata conoscenza del mondo) e cause legate alla struttura del discorso (problemi nell'organizzare informazioni in un testo coerente). Inoltre, è necessario sottolineare che alla base di un errore ci possono essere più cause.

Le cause psicolinguistiche, quelle maggiormente studiate e prese in esame in questo contributo, si dividono in cause interlinguistiche (gli errori sono causati da un transfer negativo/dall'interferenza con la L1 o altre LS che l'apprendente conosce o studia) e in cause intralinguistiche (gli errori sono dovuti a un incompleto apprendimento delle regole della LS e/o alla ipergeneralizzazione di queste regole, ecc.).

Cattana e Nesci (2004: 81 e 82) menzionano varie cause di errori in una LS, precisando che spesso è difficile risalire alla vera causa di un determinato errore (e soprattutto individuarne una sola). Gli errori sono dovuti:

- alla lingua madre;

- ad un'altra lingua straniera conosciuta, specialmente nel caso in cui la L1 dell'apprendente è molto diversa dalla LS, mentre l'altra LS che conosce è, invece, affine;

- alla lingua da apprendere da cui provengono errori di ipergeneralizzazione, errori di semplificazione, errori di regolarizzazione, errori di commistione delle strutture, ecc., che sono legati a vari stadi dell'interlingua individuale;

- alle strategie di comunicazione e di apprendimento;

- ad altre cause (tra cui i fattori personali, il metodo di insegnamento, il luogo in cui si studia, la cultura di origine, ecc.). 


\subsection{Correzione degli errori}

Sebbene la correzione degli errori abbia anche degli svantaggi (può essere fonte di frustrazione, insicurezza e uno degli ostacoli per la spontaneità nell'uso della lingua target) non dobbiamo sottovalutare il fatto che gli errori possono strutturarsi in un'abitudine negativa: se l'apprendente continua a ripeterli e non viene corretto è probabile che si fossilizzino (cf. Long 2003:513; Mezzadri 2015: 271). Pertanto, la correzione è uno dei fattori che impedisce la fossilizzazione degli errori e permette all'apprendente di raggiungere i più alti livelli di competenza linguistica in una LS. Inoltre, essa rende possibile, la riflessione linguistica che consente allo studente di verificare le ipotesi formulate sulla lingua, ma solo nel caso in cui abbia ricevuto un feedback correttivo appropriato. La correzione è quindi necessaria, e un apprendente che abbia serie intenzioni di migliorare la sua competenza nella lingua target si aspetta che essa venga fatta dal suo insegnante. Ciononostante, va ribadito che la correzione deve essere adattata al tipo di attività, all'apprendente e al suo livello. Molti studi (riportati in Pawlak 2014: 73) che hanno voluto dimostrare l'(in)efficienza della correzione della produzione orale e scritta hanno confermato che la correzione ha effetti positivi nel processo di apprendimento di una LS. Hartshorn e Evans, così come altri autori (riportati in Hartshorn e Evans 2015: 12-14 e 28) hanno dimostrato che la correzione di compiti scritti può migliorare l'accuratezza linguistica degli apprendenti, mentre non influisce su altri aspetti come la fluenza o scioltezza nell'esprimersi, la complessità del discorso dell'apprendente, ecc).

Si deve però essere consapevoli anche delle limitazioni della correzione degli errori: l'analisi degli errori da sola non ci mostra necessariamente quello che l'apprendente ha appreso. Al contrario, ci porta a focalizzare l'attenzione su ciò che non ha appreso, un atteggiamento molto diffuso tra insegnanti e apprendenti che per questi ultimi non è particolarmente motivante. Pertanto gli apprendenti, avendo paura di commettere errori, evitano strutture difficili e consegnano compiti che magari sono privi di errori, ma che non rispecchiano la loro vera competenza linguistica.

Nella nostra situazione, cioè in un contesto di studio universitario di una LS, è impossibile concepire l'apprendimento senza un'appropriata correzione degli errori e senza una dettagliata riflessione linguistica e grammaticale Un apprendimento che si concentri solo sul significato, come dimostrato da vari autori (cf. Pawlak, 2014: 9), non è sufficente per raggiungere alti livelli di competenza in una LS. 


\subsection{La metodologia, lo scopo e le ipotesi}

Per l'analisi è stato costruito un learner corpus di 100 compiti scritti ${ }^{6}$ degli apprendenti, studenti slovenofoni del secondo anno del corso in Laurea Triennale di Lingua e letteratura italiana che hanno frequentato il corso Italijanske lektorske vaje $2^{7}$ (in italiano: Esercitazioni di lingua italiana 2). $\mathrm{Nel}$ corpus sono stati inclusi testi di varie tipologie, tra cui temi espositivi, temi argomentativi, testi narrativi, riassunti, recensioni e testi scritti su testi modello. È parso utile includere tipologie diverse di testi, poiché ogni tipologia esige, almeno in parte, l'uso di mezzi linguistici diversi. Questo ci ha quindi permesso di trovare un numero più diversificato di errori. Anche se per obiettivi didattici si è optato per una correzione mista di correzione rilevativa e di correzione classificatoria ${ }^{8}$ come aiuto/sostegno per l'autocorrezione ${ }^{9}$ da parte degli studenti, per la presente analisi i compiti sono stati corretti una seconda volta e analizzati seguendo il metodo di analisi degli errori proposto da Ellis e descritto in Saville-Troike (2006: 39-40):

- costruzione del corpus,

- identificazione dell'errore,

- descrizione dell'errore ${ }^{10}$,

- $\quad$ spiegazione dell'errore,

- $\quad$ valutazione dell'errore ${ }^{11}$.

È stata eseguita un'accurata analisi dei compiti scritti al fine di presentare e spiegare i più tipici e frequenti errori degli apprendenti slovenofoni in italiano e di poter sfruttare detta analisi per progettare il futuro insegnamento e apprendimento dell'italiano come LS nella nostra facoltà, e in modo particolare per progettare (e valutare) i corsi Italijanske

6 I compiti sono stati raccolti negli anni accademici 2014/2015, 2015/2016 e 2016/2017.

7 Il corso è previsto dal piano di studi al secondo anno degli studi di Lingua e letteratura italiana nella Facoltà di Lettere e Filosofia dell'Università di Lubiana ed è pensato per gli studenti che hanno già raggiunto il livello B2 del QCER (2002), ma in realtà vi partecipano studenti, il cui livello va dall'A2+/B1 verso il C1 (rarissimi gli studenti di livello $\mathrm{C} 1$ ). La situazione è dovuta al fatto che molti dei nostri studenti negli ultimi anni iniziano gli studi come principianti assoluti o al livello A1. Si tratta di un corso pratico il cui fine è praticare le quattro abilità linguistiche (leggere, ascoltare, parlare e scrivere) e conoscere alcuni aspetti culturali dell'Italia. Per il corso si usa il libro di testo Nuovo Contatto $\mathrm{Cl}$ più alcuni altri materiali, preparati dal docente.

8 La funzione della correzione rilevativa è di contrassegnare gli errori senza però dare all'apprendente altre indicazioni. La correzione classificatoria è più dettagliata, ma comunque vengono date allo studente solo informazioni sul tipo di errore (Cattana e Nesci 2004:) e non la forma/variante corretta.

9 Ogni studente deve correggere il compito seguendo i segni (le correzioni) del docente. La decisione di usare un sistema di segni aiuta e motiva gli studenti dato che li aiuta a pensare agli errori e correggerli da soli se ne sono capaci (Harmer 2004, in Pawlak 2014: 145).

10 La descrizione è stata fatta in base alle strategie superficiali di confronto dell'enunciato prodotto dall'apprendente con l'enunciato di un parlante madrelingua, tra cui: la scelta di un elemento non previsto dal sistema, l'omissione cioè l'assenza di un elemento, l'aggiunta di un elemento, la malformazione di un elemento e l'ordine improprio degli elementi (Cattana e Nesci 2004:72-75).

11 L'ultima parte è stata esclusa poiché non rientra nella tematica del presente contributo. 
lektorske vaje 1 e 2. Solo con un' analisi approfondita degli errori nella produzione (scritta e orale) sarà possibile preparare le lezioni in modo da dare più spazio alle strutture grammaticali, sintattiche e lessicali che sono fonte di maggiori problemi. In questo modo sarà possibile offrire agli studenti un sostegno per affrontare gli errori sistematici non ancora fossilizzati. Infine, una tale analisi ci permetterà di scoprire le interferenze della L1 e di alcune altre LS e forse altre cause degli errori che gli studenti commettono nell'italiano come LS.

Si ipotizza che, pur avendo studiato in dettaglio le regole più importanti della morfologia e della sintassi, molti studenti non sono ancora in grado di applicarle/usarle correttamente nella produzione scritta ${ }^{12}$. Si ipotizza, inoltre, che la maggioranza degli errori che gli studenti commettono possono essere riconducibili a tre fonti:

- alla L1 (quindi allo sloveno),

- ad altre lingue che i nostri discenti studiano o conoscono ${ }^{13}$,

- alla lingua da apprendere (in questo caso l'italiano) cioè agli aspetti particolari di questa lingua che possono essere molto complessi.

\subsection{Risultati e discussione}

A causa dell'ampiezza dell'argomento trattato verranno presentati solamente gli errori più frequenti in base alla categoria linguistica (includendo i più frequenti errori sia morfologici che sintattici), seguiti alla fine anche dagli errori lessicali. Ogni errore sarà illustrato con esempi presi dal nostro corpus ${ }^{14}$. A volte viene presentato anche il numero di errori di un certo tipo non tanto per essere interpretato in modo assoluto ma per dare un'impressione quantitativa sull'estensione del problema, e per scoprire le differenze tra alcuni errori (come per esempio l'aggiunta errata dell'articolo determinativo confrontata con l'aggiunta errata dell'articolo indeterminativo-cf. par. 3.2.2) e come un dato aggiuntivo con cui illustrare e sostenere l'interpretazione.

\subsubsection{Ortografia}

Nei cento temi analizzati sono stati trovati 154 errori di ortografia, tra cui i più frequenti sono: l'omissione dell'accento grafico ( 74 errori), la semplificazione delle consonanti geminate (34 errori) e le parole scritte in modo sbagliato (22 errori). Va subito precisato

12 È possibile ipotizzare la stessa cosa per la produzione orale, che però non è oggetto del presente contributo.

13 L'interferenza si può verificare sia tra lingue molto diverse sia tra lingue affini. Le ultime, proprio per la loro somiglianza, spesso rappresentano una difficoltà persino maggiore per l'apprendente e diventano una secondaria fonte di errori nella LS, anche se, paradossalmente, specialmente ai livelli elementari, le somiglianze agevolano l'apprendimento e la comunicazione.

14 Per mancanza di spazio non verranno trascritte sempre frasi intere ma il contesto sufficiente per capire l'errore. Anche se in alcuni esempi è possibile trovare più errori, saranno segnati e analizzati solo quelli di nostro interesse nel dato contesto. 
che il numero di errori non rispecchia la situazione reale, visto che la maggioranza degli studenti consegna i temi scritti al computer e usa correttori automatici ${ }^{15}$. Fatto che viene confermato proprio dalla presenza di errori che un correttore automatico non è in grado di segnalare. Uno degli esempi tipici sono i monosillabici su cui bisogna segnare l'accento grafico per non confonderli con gli omografi (cf. Serianni, 2010: 56 - 57). Tra le 74 omissioni di accento grafico ben 18 volte vengono scambiate la congiunzione $e$ e la voce del verbo essere è (esempi $1-5$ ) e sei volte il pronome tonico sé e il pronome atono o la congiunzione se (esempi 6 e 7). Gli studenti, nella maggior parte di questi casi, omettono l'accento grafico.

(1) *e entrata (2) *e molto arrabbiata (3) *e molto complicato (4) *è proprio questo è in fascino, (5) *e una narrazione (6) con *seporta (7) sicura di *se

Un altro tipo di errore molto frequente e sistematico per alcuni studenti è l'omissione dell'accento grafico sui polisillabici tronchi su cui l'accento grafico deve essere segnato (cf. Serianni, 2010: 56 - 57). L'errore si verifica spesso anche quando si tratta di parole di uso frequente come così, perché, però, ecc. $(8-10)$.

(8) *citta (9) *difficolta, (10) *eta

Per gli apprendenti slovenofoni è molto tipica la semplificazione delle consonanti geminate $(11-13)$, errore che si presenta ancora più ricorrente quando gli studenti non usano il correttore ortografico. Solo poche volte vengono invece raddoppiate consonanti che non dovrebbero esserlo, quasi sicuramente a causa dell'ipercorrezione $(13-14)$. Nell'esempio (13) ci sono addirittura due errori in una sola parola.

\section{(11) *descrito (12) *opure (13) *sociallizare (14) *gommito}

Sono stati scoperti anche alcuni casi di sostituzione dell'apostrofo con l'accento (15), errore osservato esclusivamente nell'espressione un po'. È un errore fatto da pochi studenti ma per quelli sembra essere sistematico.

\section{(15) $u n^{*}$ pò delusa}

Va menzionata a questo punto anche la punteggiatura, che andrebbe analizzata più in dettaglio; per mancanza di spazio, tuttavia, ci limitiamo a dare solo alcune osservazioni generali.

15 Gli studenti possono consegnare i lavori scritti a mano o al computer. Quando scrivono al computer gli viene consigliato di non usare il correttore automatico per la prima versione. 
I nostri studenti usano nella stragrande maggioranza dei casi solamente tre segni interpuntivi: il punto, la virgola e la parentesi. Raramente appare il punto interrogativo (fatto dovuto probabilmente alla tipologia dei testi assegnati). Gli altri segni sono usati raramente e quasi sempre in modo sbagliato. Questo fenomeno è facilmente spiegabile come conseguenza del fatto che gli studenti non abbiano ancora trattato in modo sistematico e dettagliato le funzioni e gli usi dei segni interpuntivi. Li usano, pertanto, aiutandosi con la L1, il che risulta spesso sbagliato poiché le regole d'uso della punteggiatura sono diverse nelle due lingue e molto più rigide in sloveno che in italiano. Uno dei tipici errori (che si presenta come sistematico) è l'uso della virgola dopo alcune congiunzioni e pronomi relativi che introducono frasi dipendenti, casi in cui in sloveno è obbligatoria la virgola, in italiano invece di regola non viene usata. Gli esempi più caratteristici sono senza dubbio le completive introdotte dalla congiunzione che (16) e le relative limitative introdotte dal pronome relativo che (17).

(16) Ma nessuno sa*, che sotto la terra c'è uno "gnomo”... (17) Molti studenti*, che sono alle prese con le pressioni da superare,...

\subsubsection{Articolo}

Nel nostro corpus sono stati trovati 397 errori di forma ed errori nell'uso dell'articolo. Abbiamo incluso in questa parte anche le preposizioni articolate, limitandoci tuttavia a prendere in considerazione soltanto i casi di quelle preposizioni articolate in cui è l'articolo la parte sbagliata della preposizione articolata. Già Raymóndez Fernandéz e Santiago Alonso (2004: 17) con l'analisi degli errori in spagnolo hanno scoperto che l'uso dell'articolo è uno dei problemi più comuni e diffusi per gli apprendenti slovenofoni, dato che in sloveno l'articolo non esiste e l'(in)determinatezza viene espressa da meccanismi linguistici diversi da quelli dello spagnolo. L'articolo si è rivelato uno dei problemi principali anche per gli apprendenti slovenofoni del francese (cf. Žugelj 2005: 101; Lah 2006: 32) e dell'italiano (come dimostrano i risultati nel presente contributo).

Tra gli errori più frequenti nell'uso dell'articolo determinativo (AD), dell'articolo indeterminativo (AI) e dell'articolo partitivo (AP) dei nostri studenti sono:

- l'omissione dell'articolo (117 errori);

- la sostituzione dell'AI o dell'AP con l'AD (93 errori). Sono stati, invece, indentificati pochissimi errori di sostituzione "in direzione opposta";

- l'aggiunta errata dell'AD nei casi in cui non serve (68 errori). Poche volte, invece, viene aggiunto l'AI;

- $\quad$ la forma sbagliata dell'articolo (51 errori);

- gli errori d'uso dell'articolo con gli aggettivi possessivi e dimostrativi (40 errori).

Le tre forme che si sono dimostrate come le più problematiche sono: l'AD l', l'AD gli e l'AI un' (18-24). Pare che i nostri studenti non abbiano ancora interiorizzato alcune 
regole dell'uso delle forme degli articoli. Usano, infatti, soltanto un limitato numero di forme che estendono anche a quelle più "problematiche", semplificando così un sistema abbastanza complesso: usano così la o $i l$ al posto di $l$ ', $i$ al posto di gli e $u n$ al posto di $u n$ '. Serianni (2010: 163) menziona dei cambiamenti nell'uso dell'elisione di la e una. Anche se l'elisione sia della prima che della seconda forma è raccomandabile, spesso troviamo, specialmente nella stampa, esempi di mancata elisione. L'elisione di lo davanti a vocale (quindi per il maschile), invece, è da considerarsi obbligatoria. Negli esempi che seguono ci sono, quindi, delle forme che alcuni potrebbero valutare come corrette (18 e 20), ma siccome tutte le grammatiche che usiamo riportano e consigliano l'elisione, abbiamo deciso di seguire nella correzione proprio queste regole ${ }^{16}$.

(18) *la opzione (19) *il armadio (20)occuparsi *della organizzazione (21) un ora prima *del esame

(22) *un ossessione (23) *una esperienza (24) *i annunci (25) uno *dei artisti (26) gran parte *dei studenti

Il più frequente errore nell'uso dell'articolo degli apprendenti slovenofoni è l'omissione dell'articolo $(27-31)$, anche nei casi in cui dovrebbe far parte della preposizione articolata $(32-35)^{17}$.

(27) che ha fatto *? cosa molto grande (28) ruba delle cose da mangiare e anche *? forno giocattolo (29) sono *? veri e propri manipolatori (30) ... aiutarli a prendere *? decisioni (31) nessuno ha*? diritto di togliercelo (32) arriva $a *$ ? suo posto (33) dipende solo da*? persona (34) interessata a*? psicologia (35) l'essenza di*? amicizia

Come si può evincere dagli esempi, si tratta di casi molto evidenti, nei quali un parlante madrelingua o un apprendente a un livello alto di competenza linguistica avrebbe messo l'articolo. È uno degli errori tipici che possiamo in parte attribuire alla L1, poiché lo sloveno è una lingua in cui l'articolo come parte del discorso non esiste. Trenkić (2009: 5), che ha studiato lo stesso fenomeno, cioè l'omissione dell'articolo nell'inglese LS, è del parere che questo elemento venga omesso in molti casi perché sembra non essere necessario per l'aspetto comunicativo (il messaggio viene trasmesso comunque) ed è, quindi, dal punto di vista pragmatico, superfluo. La ragione per l'omissione dell'articolo va cercata anche nel fatto che la funzione primaria dell'articolo non sarebbe quella di esprimere (in)determinatezza, ma di facilitare l'elaborazione della grammatica segnalando il gruppo nominale (ibid. 6). Nel caso dello sloveno, in cui

16 Gli studenti sono a conoscenza di questa decisione.

17 Il punto interrogativo negli esempi viene usato in sostituzione dell'elemento omesso. 
l'articolo non esiste, l'(in)determinatezza del nome viene interpretata dal contesto (cf. Premrl, 2016).

Ci sono molti errori di sostituzione di un tipo di articolo con l'altro. È stato scoperto un sovrauso dell'AD a scapito dell'AI e dell'AP (36 - 39). Pochissimi sono gli esempi di sostituzione errata dell'AD con l'AI o l'AP.

(36) comincia a comprare *le cose (37) ha *gli occhi bellissimi (38) hanno *i motivi (39) il fatto che una persona si ponga ossia abbia *i obiettivi

Frequentemente i nostri studenti aggiungono l'articolo dove nella lingua target non si usa. Spesso è così, forse, a causa della regolarizzazione: gli studenti, infatti, sono consapevoli del fatto che in italiano devono usare l'articolo davanti a un nome, ma non hanno interiorizzato, e quindi non usano nella loro produzione linguistica, quelle regole che si riferiscono ai casi in cui l'articolo di regola viene omesso. Analizzando gli esempi diventa chiaro che l'aggiunta dell'articolo avviene spesso proprio nelle unità fraseologiche ${ }^{18}$, quindi in alcune espressioni prefabbricate che frequentemente non accettano modificazioni o inserimenti $(40-42)$; per questa ragione l'apprendente le deve imparare (per le unità fraseologiche cf. par. 3.2.5 e 3.2.7).

(40) fare *lo sport (41) grazie *al Dio (42) Guido li trattava con *un estrema attenzione.

Gli aggettivi dimostrativi e l'articolo determinativo si escludono a vicenda. Ma gli studenti, che tendono a regolarizzare la lingua, applicano la regola generale; aggiungono quindi l'AD davanti all'aggettivo (dimostrativo) (43 e 44).

\section{(43) *al questo punto (44) *diverso dal quello vero}

Un altro errore sistematico sembra l'uso sbagliato dell'articolo con gli aggettivi possessivi (APos). In Italiano, per regola generale, davanti agli APos si usa l'articolo. Gli errori in questo caso sono due: l'omissione dell'articolo, che più che per il transfer negativo dalla L1 è causata da altre lingue (in questo caso spagnolo, francese e forse inglese, lingue in cui non si mette l'articolo davanti agli APos), dato che quasi tutti gli errori sono stati fatti da studenti che studiano o francese o spagnolo, ma non da altri studenti (45 e 46). Inoltre, gli studenti hanno molti problemi con le poche eccezioni legate all'uso dell'APos, ovvero con i casi in cui l'APos precede un nome di parentela. In alcuni casi, difatti, l'articolo andrebbe omesso, ma gli studenti lo aggiungono, applicando un'altra volta la regola generale (47 e 48).

18 Nel nostro caso si tratta di collocazioni e locuzioni. 
(45) *era *? suo ragazzo (46) per *? nostro studio (47) la storia *del suo figlio (48) *La sua madre già da bambina...

\subsubsection{Nome}

Nel corpus sono stati trovati per questa categoria 51 errori.

Alcuni studenti dimostrano di avere non pochi problemi nel determinare il genere dei nomi, errore verificatosi specialmente nei casi in cui il genere di un sostantivo in italiano e in sloveno non coincidono. Sono questi i casi in cui, spesso, gli apprendenti si aiutano con la L1 e attribuiscono ai sostantivi italiani il genere che questi hanno in sloveno. Si noti che negli esempi (52) e (55) lo studente ha adattato anche la desinenza dei due nomi (si tratta di una malformazione).

(49) *una gruppo (50) *il mio opinione (51) *i parti, il parte (52) *il ricetto (53) *un riunione (54) *questo ragioni (55) *un risposto giusto

Sono state trovate anche altre malformazioni di nomi maschili che terminano in $-a$ (56-57)

\section{(56) *il sistemo $(57) * i l$ problemo}

Inoltre, spesso gli studenti sbagliano il genere del nome perché trattano le eccezioni conformemente alla regola generale, che in questo caso è "i nomi femminili terminano in $-a$ e i nomi maschili in $-O$ " (Serianni 2010: 116 e 117).

(58) *sul radio (59) è *una tema complessa (60) per *i nostri star

Nell'esempio (60) è chiaro che la regola "sono inoltre quasi tutti maschili i nomi, perlopiù di origine straniera, terminanti in consonante” (Serianni 2010: 110) è stata interiorizzata. Comunque, anche questa regola ha delle eccezioni di genere femminile come la star.

Infine, gli studenti spesso usano la forma sbagliata del plurale. Nei tre casi esposti (61 - 63) la forma è sbagliata per vari motivi: la regolarizzazione (61), l'interferenza della desinenza della preposizione articolata e il fatto che la $-e$ è una delle desinenze possibili per il plurale in italiano. Non è stato possibile trovare la causa linguistica alla base dell' errore (63).

(61) $i$ *roboti (62) sulle *rete sociali (63) gli *esame 


\subsubsection{Aggettivo}

Sono stati trovati 80 errori nella forma e nell'uso dell'aggettivo, di cui saranno menzionati, di seguito, quelli legati agli aggettivi qualificativi e agli aggettivi possessivi, poiché si sono dimostrati i più frequenti e sistematici.

Per quanto riguarda l'aggettivo qualificativo sono più frequenti gli errori di accordo dell'aggettivo qualificativo con il nome, per i quali a volte è difficile stabilire le cause linguistiche. Negli esempi $(64,67$ e 68) gli errori sono probabilmente attribuibili alle desinenze dei nomi e degli aggettivi che compaiono nello stesso sintagma. L'apprendente usa erroneamente per l'aggettivo la stessa desinenza che per il nome (64 e 68) o la stessa desinenza di un altro aggettivo presente nel sintagma (67).

(64) caratteristiche *difficile da (65) ossessione *morboso (66) occhi *marrone (67) sentimento forte e *profonde (68) *tanti decorazioni

Un altro problema ricorrente degli apprendenti slovenofoni è la posizione sbagliata dell'aggettivo qualificativo. Serianni (2010: 200) spiega che "la posizione non marcata dell'aggettivo qualificativo è dopo il nome cui si riferisce". Se invece l'aggettivo precede il nome "indica una maggiore soggettività di giudizio in chi parla o scrive". Lo spostamento dell'aggettivo in alcuni casi comporta un radicale cambiamento di significato ${ }^{19}$. In sloveno, la posizione dell'aggettivo qualificativo in funzione di attributo è sempre prima del nome. Crediamo che sia proprio la differenza tra le due lingue a spingere gli studenti ad usare spesso l'aggettivo qualificativo prima del nome anche nei casi in cui in italiano andrebbe messo dopo (69-72).

(69) è presente in molti *importanti paesi (70) le *famose persone (71) hanno una *migliore capacità (72) la lingua *unica

Inoltre, gli apprendenti spesso confondono alcuni aggettivi qualificativi con avverbi di modo, errore che appare particolarmente ricorrente per le seguenti coppie: buono vs. bene, cattivo vs. male, migliore vs. meglio, peggiore vs. peggio ecc.

(73) questo * male esempio (74) è molto * bene per (75) qualsiasi pubblicità anche se *male (76) creare un *meglio mondo

Per quello che riguarda gli aggettivi possessivi, oltre agli errori legati all'uso dell'articolo con gli aggettivi possessivi (cf. 45 - 48), si è rivelata molto frequente la sostituzione dell'aggettivo possessivo loro con suo/sua. Sono per lo più gli studenti di spagnolo a fare questo tipo di errore siccome in spagnolo l'aggettivo possessivo su si usa sia per la

19 Per altre regole sulla posizione dell'aggettivo in italiano cf. Serianni (2010: 199 - 205). 
terza persona singolare che per la terza persona plurale. In italiano, invece, esistono due forme diverse, suo per il singolare e loro per il plurale.

(77) non avevano problemi perché $i$ *suoi genitori (78) i giovani e le *sue vacanze (79) $i$ giovani si prendono cura dei * suoi profili

\subsubsection{Pronome}

Sono stati trovati circa 180 errori legati alle forme e agli usi sbagliati di diversi tipi di pronomi.

L'errore sistematico e molto frequente, legato all'uso dei pronomi personali atoni, è la sostituzione dei pronomi diretti (PD) con i pronomi indiretti (PI) e la sostituzione dei PI con i PD ${ }^{20}$, di cui di seguito presentiamo alcuni esempi:

a) la sostituzione del PI con il PD con i seguenti verbi: interessare, chiedere, dare, dire, regalare, ordinare. Sono stati fatti particolarmente molti errori con i primi due verbi menzionati. Quasi in tutti i casi si tratta di verbi che possono reggere sia il complemento oggetto che il complemento di termine, quindi possiamo usare con questi verbi sia i PD che i PI. L'uso dell'uno o dell'altro dipende dal contesto.

(80) l'intervistatore *la chiede di (81) il regalo non *lo interessa (82) *lo ha dato una moneta (83) *li disse di smetterla

b) la sostituzione del PD con il PI, specialmente con i seguenti verbi: aiutare, conoscere, domandare, disturbare, ordinare, portare, unire, fare,... Sebbene alcuni di questi verbi ammettano tutte e due le funzioni, ci sono nella lista anche verbi che ammettono solo il complemento oggetto (in grassetto).

(84) nessuno *gli conosce $(85) *$ gli invidio (86) si accorge che qualcuno *gli sta seguendo (87) nessuno *gli disturba (88) ha deciso di *aiutargli (89) conoscenze che possono *aiutargli

È possibile attribuire con certezza l'uso sbagliato del PI atono con il verbo aiutare (88 - 89) alla L1. In sloveno, infatti, il verbo aiutare non ha la stessa reggenza che in italiano e gli apprendenti sistematicamente usano questo verbo con i PI o la preposizione seguita da un nome. Negli altri casi la reggenza nella L1 e nella LS è la stessa quindi è difficile stabilire la causa degli errori.

20 In tutti i casi si tratta di pronomi diretti nella $3^{\mathrm{a}}$ persona singolare e plurale. Nella $1^{\mathrm{a}} \mathrm{e}$ nella $2^{\mathrm{a}}$ persona i pronomi diretti e indiretti atoni hanno delle forme uguali quindi è stato impossibile determinare se gli apprendenti abbiano capito se sono in funzione di un complemento oggetto o di un complemento di termine. 
Al contrario della frequenza con cui si manifestano gli errori nell'uso dei PD e dei PI è stato possibile scoprire pochissimi (solamente due) errori legati ai pronomi combinati. Questo dato sorprendente non dipende dal fatto che gli studenti sappiano usare bene i pronomi combinati ${ }^{21}$ ma dal fatto che evitino di usarli proprio per paura di sbagliare. Invece di riprendere un sostantivo con un pronome lo ripetono o usano qualche altro mezzo per farlo, come per esempio i dimostrativi questo e quello. Un'ulteriore causa della bassa frequenza dei pronomi combinati potrebbe essere la tipologia dei testi.

In alcuni casi gli studenti omettono il pronome credendo che non sia necessario. Quando, per esempio, in una frase abbiamo la dislocazione dell'oggetto diretto a sinistra, questo deve essere ripreso mediante un pronome atono. In costrutti del genere gli studenti fanno errori sistematici $(90-92)$. Da una parte la causa è sicuramente l'ordine atipico della struttura sintattica e dall'altra il fatto che nella L1 un pronome in casi analoghi non viene usato poiché superfluo ${ }^{22}$.

(90) L'ultimo viaggio *? ho fatto il fine di settembre (91) le cose che ci interessano*? leggiamo volentieri (92) questo*? sentiamo ogni giorno

A volte invece di riprendere il nome con il pronome gli studenti lo omettono, come negli esempi (93 - 95), oppure ripetono il nome una seconda volta (96).

(93) Se si promette qualcosa e poi non *? si realizza (94) gli lascia il suo numero perché lui possa avvertire*? quando finisce (95) mi ha chiesto cosa fosse successo e *? ho spiegato che (96) Il cane cerca di attirare l'attenzione del ragazzo. Trova una palla e la porta al ragazzo.

Come ultimo errore legato all'uso dei pronomi, deve essere menzionata l'omissione del pronome dimostrativo quello in combinazione con tutto nell'espressione tutto quello + dipendete relativa. Nella maggioranza dei casi, per l'interferenza dello sloveno (L1), gli apprendenti omettono il dimostrativo quello, elemento necessario in italiano, ma spesso omesso in sloveno $(97-99)$. Inoltre, è frequente la sostituzione del dimostrativo questo con quello (100).

(97) ha già detto tutto*? che si può (98) tutto *? che faceva (99) tutto *? che fanno (100) parlano di *questo che gli succede

21 Durante le lezioni è possibile osservare un mancato uso dei pronomi combinati. Quando, però, gli studenti li usano fanno molti sbagli. Inoltre, si sentono confusi e frustrati se obbligati ad usarli.

22 In alcuni casi la struttura sintattica in L1 cambia del tutto. 


\subsubsection{Preposizione}

Tra gli elementi della lingua italiana la preposizione è, probabilmente, uno dei più difficili da apprendere, proprio per le enormi differenze tra lo sloveno e l'italiano. Mentre lo sloveno ha i casi ed esprime alcuni rapporti sintattici attraverso la declinazione, in italiano, una lingua in cui il caso morfologico non esiste, si esprimono gli stessi rapporti con le preposizioni. Proprio per questa ragione una preposizione in italiano ha più funzioni di una preposizione in sloveno. Widlak (in Sosnowski 2010: 148), che ha messo a confronto un testo italiano e la sua traduzione in polacco (lingua slava come lo sloveno), ha scoperto che oltre la metà delle preposizioni italiane nella traduzione viene espressa attraverso la declinazione. Nel nostro corpus sono stati trovati 228 usi sbagliati di preposizione.

Uno degli errori più frequenti è l'aggiunta errata delle preposizioni $d i$ o $a$ nelle espressioni impersonali composte da essere + aggettivo/avverbio, seguite da un infinto (101 - 105). Gli apprendenti tendono ad aggiungere la preposizione tra l'aggettivo e la dipendente soggettiva implicita, caso in cui l'italiano non richiede la preposizione (cf. Serianni 2010: 566). In sloveno in questi casi al posto dell'aggettivo o dell'avverbio si trova prislovni povedkovnik che è seguito nella maggioranza dei casi da un infinito (Žele 2009: 67). Pertanto gli errori non possono essere spiegati con l'interferenza della L1. Questo errore è, forse, attribuibile al fatto che in italiano molte dipendenti implicite con l'infinito vengano introdotte proprio da una preposizione, anche se, come si vedrà più tardi, spesso gli apprendenti slovenofoni proprio in questi casi (cioè prima delle implicite) omettono la preposizione.

(101) è difficile * a promuovere (102) non è sempre facile *a rispondere (103) è importante *di scegliere (104) penso che sia meglio *di lavorare (105) è necessario *di seguire

Molti sono anche gli errori di reggenza di preposizioni con il verbo come elemento reggente. Di questa problematica viene fatta menzione e spiegate le cause nell'Enciclopedia dell'italiano Treccani (2010):

"La selezione di una preposizione da parte di un elemento reggente è per lo più un fatto formale, arbitrario o dovuto a fenomeni storici. In effetti, costituisce uno degli aspetti più complicati dell'apprendimento di una lingua, sia da parte dei bambini che da parte degli stranieri."

Proprio per quest'arbitrarietà ${ }^{23}$, caratteristica della LS che studiano, forse ci sono tanti errori di sostituzione di una preposizione con un'altra. Analizzando gli esempi del

23 Negli schemi di reggenza è comunque possibile trovare anche delle regolarità. La stessa preposizione può occorrere in reggenze semanticamente o sintatticamente simili. Inoltre, lo stesso elemento reggente può reggere più preposizioni. 
nostro corpus non è stato possibile stabilire delle regolarità; ci sono, tuttavia, dei verbi con cui gli errori sono più frequenti (106-110).

(106) ho cominciato *di insegnare (107) telefoni iniziano *di suonare (108) abbiamo imparato *di leggere (109) pensare * su questo argomento $(110)$ pensiamo *di qualcosa di scandaloso

Un altro errore verificatosi con frequenza è l'omissione della preposizione davanti alle implicite oggettive $(111-115)$. Spesso gli apprendenti commettono questi errori per l'interferenza della L1, in cui il verbo nella forma personale viene seguito direttamente da un infinito o, in alcuni casi, da una dipendente esplicita.

(111) ho finalmente iniziato *? correre (112) comincia *? comprare (113) gli aveva permesso *? vedere (114) ho tentato *? parlare con la signora (115) utili per aiutarmi $*$ ? capire

Nei compiti scritti degli studenti che studiano/conoscono lo spagnolo sono stati trovati molti esempi di errore in cui per l'interferenza con questa lingua il complemento oggetto viene introdotto dalla preposizione $a$, preposizione che in italiano introduce il complemento di temine (e alcuni altri complementi, ma mai il complemento oggetto). In spagnolo, infatti, la preposizione $a$ può introdurre un complemento oggetto nel caso in cui questa funzione sia svolta da un nome di persona o animale o un sostantivo animato (116 - 120). Per l'esempio (120) è possibile sia l'interferenza dello sloveno (dativo) sia dello spagnolo (agradecer a alguien).

(116) aspettando * a Scarlet (117) incolpa *ai genitori (118) sa torturare *ai spettatori (119) un ragazzo sta aspettando *a una ragazza (120) ringrazia *ai genitori

Va aggiunta un'altra osservazione interessante, ossia la sostituzione della preposizione $d a$ con $d i$ da parte degli studenti di spagnolo e di francese. In queste due lingue la preposizione $d a$ non esiste, quindi rappresenta per gli apprendenti dell'italiano un elemento nuovo e aggiuntivo che, per l'interferenza delle altre lingue straniere che conoscono, semplificano usando la preposizione $d i$ al posto di $d a(121-123)$.

(121) *di dove vieni (122) possiamo imparare *di loro (123) riceve un messaggio *di qualcuno

Sono stati trovati altri errori nell'uso delle preposizioni riconducibili all'interferenza della L1 o di un'altra LS che gli apprendenti studiano/conoscono. 
(124) si è innamorata *in questo cappotto - sloveno (125) aiutare *alle persone in difficoltà - sloveno (126) l'intelligenza l'ha presa *dopo il padre - sloveno (127) se non sono insieme *con qualcuno - sloveno (128) possiamo ringraziare *alla TV - sloveno (129) *di un modo più semplice - spagnolo (130) vanno *di vacanza - spagnolo

Le preposizioni sono, oltre all'articolo (cf. par. 3.2.2), un altro elemento delle unità fraseologiche (UF) ${ }^{24}$ che spesso sono fonte di errore. Gli apprendenti creano UF inesistenti, sostituendo o omettendo la preposizione.

(131) molte cose *? comune (avere in comune) (132) *da questo modo (in questo modo) (133) in mezzo *di nulla (in mezzo al nulla)

\subsubsection{Avverbio}

Sono stati trovati 41 errori nell'uso dell'avverbio, tra cui vanno messi in rilievo per la loro frequenza i seguenti:

a) l'uso dell'aggettivo al posto dell'avverbio. Anche se in italiano è possibile usare l'aggettivo in funzione avverbiale questo uso non sempre è accettabile come negli esempi (134 e 135) (cf. anche par. 3.2.4).

(134) non poteva iniziare più *sfortunato (135) per fare la spesa più * comodo

b) la maggioranza degli errori nell'uso dell'avverbio è legata alla sua posizione. Secondo Serianni (2010: 513) le regole generali sono: l'avverbio va messo normalmente prima dell'aggettivo e dopo il verbo a cui si riferisce. Se, invece, si riferisce a un'intera frase, è mobile. ${ }^{25}$ Gli studenti slovenofoni commettono errori di collocazione di avverbi, per lo più quelli che modificano il verbo. Gli studenti optano in questi casi per il transfer dalla L1, quindi decidono la posizione a seconda della sintassi slovena $(136-141)$.

(136) *sempre ha alzato il dito (137) *sempre decido (138) cosa si può fare per *veramente mantenere (139) è questo *davvero succede (140) si * piano piano allarga (141) che *urgentemente abbiamo bisogno

24 Le UF verranno analizzate più dettagliatamente parlando degli errori lessicali (cf. par. 3.2.8).

25 Per altre regole, dettagli ed eccezioni cf. Serianni (2010: 513-514) 


\subsubsection{Verbo}

Poiché il sistema verbale italiano è molto complesso di per sé, e specialmente in comparazione con lo sloveno, va messo in risalto da principio che non sarà possibile enumerare e spiegare tutti gli errori degli apprendenti slovenofoni. Verranno esposti soltanto i più frequenti nel nostro corpus. Inoltre, questo tema è stato trattato più in dettaglio già da altri studiosi sloveni (ad esempio Miklič 1992; Miklič in Ožbot 2007; Premrl 2010). Bisogna sottolineare inoltre che i compiti scritti da cui è composto il nostro corpus non si sono dimostrati un buon campione per analizzare l'uso di tutte le forme verbali per vari motivi. Gli apprendenti posti davanti al compito di scrivere una composizione scritta di regola semplificano oltremodo un sistema verbale così complesso come quello italiano: sovrausano il presente (anche per raccontare il passato), semplificano la sintassi del periodo (cf. Mertelj 2005), usano pochissimo le dipendenti implicite, ecc. Le strategie menzionate influiscono notevolmente sugli errori trovati nel corpus e presentati in questo contributo. In aggiunta, benché composto da 100 compiti scritti, il nostro corpus sembra troppo limitato (sia per il numero di testi sia per la tipologia dei testi) per analizzare alcuni fenomeni molto complessi, tra cui sicuramente l'uso delle forme verbali in italiano.

L'analisi del verbo nel nostro corpus ci ha comunque permesso di individuare alcune tipologie di errori da considerare, per la loro frequenza e natura, sistematici. L'uso degli ausiliari nei tempi verbali composti sembra essere uno di questi. Si tratta di un errore per il quale è quasi impossibile stabilire una causa unica: da una parte abbiamo il transfer dallo sloveno che, per analogia sintattica, favorisce la scelta dell'ausiliare essere (unico ausiliare per le forme verbali composte in sloveno), dall'altra c'è la complessità dell'uso degli ausiliari in italiano. Inoltre, gli studenti di spagnolo riducono spesso l'uso degli ausiliari ad avere, dato che lo spagnolo conosce soltanto un verbo ausiliare (haber). Sono stati commessi molti errori anche nei cosiddetti casi "semplici", quelli in cui è possibile decidere per uno dei verbi ausiliari in base a una regola chiara, come per esempio l'uso dell'ausiliare avere con i verbi transitivi $(142-145)$ e l'uso dell'ausiliare essere con i verbi riflessivi (147 - 148). Anche i verbi che possono essere coniugati con entrambi gli ausiliari rappresentano un problema per gli apprendenti slovenofoni $(146,148$ - 149).

(142) con questa dichiarazione *è mostrato (143) mia moglie mi *è lasciata (144) che * sono ottenute un gran successo (145) come se *fosseincontrato (146) si *ha avvicinato (147) non si *hanno trasformati (148) non *ha migliorato (149) *aveva cresciuto (150) *ha riuscita

Un altro errore tipico degli apprendenti slovenofoni, presente sia nella produzione scritta sia in quella orale, e verificatosi anche nel nostro corpus, è l'aggiunta del pronome riflessivo a verbi che in italiano non sono mai riflessivi, mentre lo sono invece in sloveno. 
Dalla nostra analisi emergono, e sono stati ulteriormente confermati, alcuni problemi degli apprendenti slovenofoni scoperti, elencati e descritti anche dagli autori menzionati sopra (Miklič 1992; Miklič e Ožbot 2007; Premrl 2010). Miklič e Ožbot (2007: 6770) sottolineano che le difficoltà nell'uso delle forme verbali da parte degli apprendenti slovenofoni sorgono sia da un numero diverso di forme verbali a disposizione nelle due lingue, sia dai principi (l'espressione della temporalità relativa, dell'aspettualità e della modalità) in base ai quali vengono scelte le forme verbali nelle due lingue. Con la nostra analisi delle composizioni scritte è stato constatato che gli errori più frequenti nell'uso delle forme verbali sono: la scelta sbagliata tra il perfetto e l'imperfetto, la sostituzione errata del congiuntivo con l'indicativo e gli errori nell'uso del trapassato prossimo, che si presentano sia come uso del passato prossimo al posto del trapassato prossimo sia come sovrauso del trapassato prossimo, esteso ai contesti in cui non dovrebbe essere usato.

Analizzando il corpus dei compiti scritti degli apprendenti è stato possibile notare un'alta frequenza di errori per la sostituzione del passato prossimo con l'imperfetto (155 -157) e dell'imperfetto con il passato prossimo $(158-160)$.

(155) * Guardava il cassiere e *nascondeva alcune cose nella borsa. (156) *Correva nella macchina e cercava di fuggire. (157) *Manteneva uno sguardo molto nervoso e arrabbiato finché siamo arrivati a Roma. (158) Il cane ha riuscito a chiudersi nella scatola mentre * ha giocato a palla e così ha fatto ridere il ragazzo. (159) Quando ero piccola non *ho capito perché mia madre e tutti gli altri correvano. (160) Un ragazzo di diciotto anni era bersaglio delle ragazze che *hanno pensato che lui era bello, e ogni volta che vedevano il ragazzo *hanno usato la frase "Damn, Daniel!".

Il secondo tipo di errori per frequenza sono gli errori di sostituzione del congiuntivo con l'indicativo (161 - 163). Il fatto che in sloveno il congiuntivo non esista ostacola notevolmente l'apprendimento su come e quando decidere di usare questo modo in italiano, una decisione influenzata indubbiamente da molti fattori che gli apprendenti devono prendere in considerazione. Un caso di ipercorrezione nei confronti della scelta tra l'indicativo e il congiuntivo sono le sostituzioni dell'indicativo con il congiuntivo, anche se poche nel nostro corpus: gli studenti usano spesso il congiuntivo con l'avverbio forse e dopo l'espressione secondo me (164 - 165).

(161) ...come se non *abbiamo imparato nulla. (162) La medaglia olimpica è l'onore più alto che uno sportivo *può ricevere. (163) Penso che *vogliono essere famosi. (164) secondo me *debba guardare (165) forse *sia meglio fare

A proposito dell'uso del trapassato, Premrl (2010: 172) menziona le differenze nell'esprimere l'anteriorità nel passato in italiano e in sloveno. La nostra analisi conferma la sostituzione del trapassato prossimo con il passato prossimo (166-169). Si è verificata 
anche una frequente ipercorrezione: gli apprendenti usano il trapassato prossimo anche nei casi in cui non deve essere usato, sostituendo quasi sempre con questa forma verbale un passato prossimo $(170-171)$. Dato che gli studenti non vengono solamente esposti a questa forma verbale, ma viene anche studiata e ne prendono coscienza durante le lezioni, cercano di usarla, benché spesso in contesti inadeguati.

(166) Era triste perché *ha litigato con la sua ragazza. (167) Non *ho notato prima, ma proprio era vuoto. (168) Mi ha detto che suo marito *è morto poco tempo fa. (169) Non ha detto niente perché sapeva che non *è arrivata in tempo e come sempre aspettava che io scuoterò la testa e continueremo a parlare da dove *i siamo fermate la scorsa volta. (170) La situazione non è poi così grave come *l'avevi descritta. (171) Nel cortometraggio seguiamo la storia di una moneta dopo che $l$ *avevano buttato nella fontana.

\subsubsection{Errori di lessico}

Gli errori di lessico dei nostri apprendenti sono tra i più frequenti. Nel corpus ne abbiamo individuati circa 360. Sono molto diversi tra loro e attribuibili a varie cause. Il nostro obiettivo è di presentarne alcuni: i più sistematici e quelli a cui è possibile attribuire una chiara interferenza della L1 e/o di altre lingue che gli apprendenti studiano.

Una prima osservazione generale sarebbe che gli studenti nei lavori scritti frequentemente semplificano il lessico usando termini generici $(172-177)$, come per esempio cosa, questa cosa, questo, quello o un verbo generico, al posto di termini più specifici che possono esprimere meglio un determinato concetto. A volte l'uso di un termine generico è addirittura un errore. Si tratta di una tendenza che andrebbe gradualmente migliorata $\mathrm{o}$ invertita (con un feedback appropriato e anche con l'uso di dizionari) verso i livelli più alti della competenza linguistica.

(172) *dare nuove esperienze (173) *questo farà i compratori felici (meglio: rendere) (174) *ha usato il nuovo rossetto. (175) *ha fatto i contatti. (176) la fama *fatta con i soldi (177) ...come una persona può diventare famosa senza un talento o* ${ }^{*}$ cosa speciale...

Ci sono alcune coppie di parole (tra cui i verbi e i nomi sono i più frequenti) che causano non pochi problemi agli apprendenti slovenofoni poiché spesso due parole/espressioni vengono confuse e di conseguenza scambiate nella produzione scritta. Sono stati individuati errori sistematici per le seguenti coppie di parole ${ }^{26}$ :

26 Durante le lezioni e negli altri compiti si sono mostrati anche altri errori simili: gli studenti confondono anche sapere/rendersi conto, trovare/incontrare, ecc. 


\section{CONOSCERE/SAPERE}

(178) ...tutti * conosciamo che lo show (179) quelli che non * sanno la propria storia

\section{PRESENTARE/RAPPRESENTARE}

(180) il Capodanno *presenta il momento più opportuno per... (181) (parlando della pubblicità) ci *rappresenta prodotti, posti da visitare, servizi... (182) quello (cose) che le pubblicità ci *rappresentano non è vero (183) la storia *presenta il sapere, il presente la comprensione e l'avvenire la pianificazione.

\section{IMPARARE/INSEGNARE}

(184) se non altro gli ho *imparato ad usare il telefonino (185) a me, la vita ha deciso di $*$ imparare alcune cose (186) gli *impara ad apprezzare

A proposito dell'ultima coppia va precisato che questi due verbi sono particolarmente difficili da distinguere per i nostri studenti. Anche se nei compiti scritti abbiamo trovato solamente la sostituzione di insegnare con imparare, è stato possibile osservare in classe e in altri compiti che spesso sostituiscono anche imparare con insegnare. Pare che lo sloveno con la coppia di parole (na)učiti (ital. insegnare qlco. a qlcu.) vs. (na)učiti se (ital. imparare qlco.) rappresenti un ostacolo notevole. Forse in aggiunta a un feedback sotto forma di spiegazione grammaticale in luce contrastiva, come mezzo adeguato per affrontare tali problemi si potrebbero proporre dei compiti regolari di traduzione pedagogica.

A volte, gli studenti usano una parola o un'espressione esistente in un contesto inappropriato. Se nell'esempio (190) lo studente non sa che imbarcarsi può essere usato solo con nave o aereo (quindi non in tutti i contesti che significano "salire su un mezzo di trasporto"), nell'esempio (191) lo studente usa, sotto l'influenza dello sloveno, un plurale esistente in italiano che però non è adatto al contesto. Nell'esempio (192) è l'inglese a interferire nella scelta della parola, che sarebbe appropriata in inglese ma non lo è in italiano. In italiano, infatti, carattere non può sostituire personaggio.

(187) sebbene la funzione delle pubblicità sia quello di *dimostrare qualcosa in modo migliore (invece di mostrare) (188) *tirano la gente alle spese spesso inutili (invece di attirano) (189) *Davanti il periodo degli esami (invece di prima del) (190) mi sono *imbarcata sul treno (191) assume il controllo sui* cervelli (sloveno) (192) *caratteri di un libro (dall'inglese - charactes in a novel) (193) c'è una *locuzione latina (locuzione per detto, proverbio)

Un fenomeno interessante e non sconosciuto come strategia è l'uso di parole o di intere espressioni inesistenti in italiano. Spesso, per l'interferenza della L1 o di un'altra LS che conoscono, gli studenti creano nuovi vocaboli o espressioni, inesistenti in italiano e quindi inaccettabili. In alcuni casi è possibile stabilire con certezza l'origine dell'errore 
in una lingua straniera che lo studente conosce o studia (194 - 196), in altri casi le cause forse dipendono da più fattori: errata formazione della parola (197), ipergeneralizzazione (198) o regolarizzazione.

(194) alla loro *questione se ho visto (inglese - question) (195) opere che ci fanno *reazionare - (spagnolo - reaccionar) (196) *esplica il filosofo - (spagnolo explicar) (197) il suo*camminaggio è troppo interessante (198) mi ha guardato *incuriositamente

Tra gli errori lessicali, numerosissimi sono quelli appartenenti alla forma e all'uso di unità fraseologiche (UF). Le UF (collocazioni, locuzioni e paremie ${ }^{27}$ ) sono "espressioni" formate da due o più parole con delle caratteristiche tipiche: si tratta di sequenze prefabbricate che si usano come un insieme (e vanno, quindi, anche apprese come tali), sono molto frequenti nella lingua, relativamente fisse (spesso non ammettono alterazioni della forma, addizioni e omissioni di elementi), molte hanno un significato opaco. Se usate correttamente sono una valida prova di un'alta competenza linguistica e sono uno degli elementi necessari per una comunicazione efficiente e autentica con i parlanti madrelingua. Proprio a causa delle caratteristiche sopra menzionate sono fonte di moltissimi errori da parte degli apprendenti non solo ai livelli bassi, ma anche a quelli più alti della competenza linguistica. L'errore più comune degli studenti è l'uso di UF inesistenti (199 - 204), che diventano tali nel momento in cui gli apprendenti modificano o sostituiscono uno degli elementi dell'UF o le creano traducendo un' UF letteralmente dalla L1 o da qualche altra lingua conosciuta.

(199) *restare in gamba - lo studente cambia un elemento dell'UF essere in gamba che rende la locuzione inesistente dato che si può usare solo con il verbo essere o con un nome (per esempio un ragazzo in gamba)

(200) *infiammati lettori - sloveno (sloveno - goreči bralci)

(201) *si guadagna la morte - (sloveno - zaslužiti sismrt)

(202) nonostante la nostra * grandezza piccola (sloveno - majhna vlikost).

(203) *mal esempio (spagnolo - mal ejemplo)

(204) *ho tenuto l'opportunità (spagnolo - tener la oportunidad)

\section{$4 \quad$ CONCLUSIONI}

In base all'analisi degli errori dei compiti scritti si è potuto constatare che gli apprendenti, pur avendo studiato in dettaglio le regole grammaticali dell'italiano, non sono ancora capaci

27 Nell'analisi rientrano solo le collocazioni e le locuzioni dato che gli studenti non hanno usato paremie nei loro compiti scritti. 
di applicarle tutte nella loro produzione scritta. Fanno moltissimi errori partendo dalla regola generale (già interiorizzata), che applicano anche alle eccezioni. Inoltre, si aiutano spesso, nei casi in cui non hanno ancora interiorizzato le regole della LS, con la L1, con altre LS che studiano e con le strategie generali di apprendimento (generalizzazione, semplificazione, ecc.). Un problema evidente sembrano essere anche gli errori lessicali.

Per molti degli errori sistematici è stato possibile ipotizzare le cause, che sono principalmente di tre tipi: errori per l'interferenza della L1, che impone le proprie strutture e regole al livello grammaticale, sintattico e, benché in minor grado, anche lessicale; errori per l'interferenza di un'altra LS che conoscono (sono stati presentati alcuni esempi di spagnolo, francese ed inglese); errori dovuti alle caratteristiche e alla complessità della LS che studiano, quindi l'italiano (rientrano sicuramente in questo gruppo le eccezioni delle singole categorie e il complesso sistema verbale). Ci sono tuttavia molti errori che non si sono verificati sistematicamente e/o di cui non è stato possibile determinare le cause linguistiche.

Bisogna, a questo punto, menzionare alcune limitazioni del corpus e dell'analisi. Nonostante il corpus fosse composto di un numero relativamente alto di compiti scritti, si è dimostrato troppo limitato per l'analisi di alcuni fenomeni, come per esempio per l'uso delle forme verbali. Inoltre, non sono stati documentati (o lo sono stati solamente con qualche esempio) alcuni degli errori tipici degli apprendenti slovenofoni che ci saremmo aspettati di trovare in base all'esperienza nell'insegnamento. Tra questi vi sono per esempio l'uso del futuro semplice al posto del condizionale composto per esprimere il futuro nel passato, errore verificatosi nel compito di un solo studente.

Ci pare importante, infine, delineare alcune possibili utilizzazioni della nostra analisi. Come è stato già spiegato, gli studenti devono effettuare l'autocorrezione degli errori, seguendo i "suggerimenti" del docente ${ }^{28}$. Inoltre, verso la metà dell'anno accademico, quando viene raccolto un numero sufficiente di compiti scritti, gli errori più ricorrenti vengono trattati in una lezione. Il punto di partenza sono sempre esempi, presi dai compiti scritti degli apprendenti, che gli studenti devono cercare di correggere, all'inizio in gruppo o in coppia e di seguito tutti insieme; vengono ripassate alcune regole (chiedendo anche agli studenti di richiamare e spiegare da soli le regole che conoscono) e vengono fornite delle spiegazioni aggiuntive. In futuro si potrebbero introdurre altre attività legate all'analisi degli errori: ogni studente dovrebbe preparare una scheda promemoria con gli errori più frequenti (come proposto da Lah 2006: 33) con cui aiutarsi sia per scrivere che per autocorreggersi. In questo modo gli studenti diventano consapevoli dei propri errori e riflettono sul modo di correggerli, il che li aiuta a non ripetere sempre gli stessi errori e a evitare la fossilizzazione. Dal docente verranno inoltre preparate, in base all'analisi fatta, delle attività supplementari per i punti più problematici. Sarebbero sicuramente di

28 Il docente segna l'errore e il tipo di errore. Inoltre, per ogni singolo compito scritto lo studente riceve anche un breve feedback correttivo con la lista degli errori ricorrenti (per es. congiuntivo, articolo con i possessivi e presentare/ rappresentare) con cui aiuta lo studente a fare la correzione. Se l'apprendente non è capace di correggere un errore gli viene fornita dal docente la risposta corretta. 
grande aiuto le traduzioni pedagogiche o delle attività comunicative centrate sull'uso di elementi e di strutture che si sono rivelate difficili per i nostri studenti. L'analisi potrebbe essere, infine, di uso e di interesse anche per altri insegnanti che insegnano italiano ad apprendenti slovenofoni e a quegli apprendenti di italiano che hanno già raggiunto un certo livello di competenza linguistica.

\section{BIBLIOGRAFIA}

CATTANA, A./M. T. NESCI (2004) Analizzare e correggere gli errori. Perugia: Guerra Edizioni.

CORDER, S. P. (1967) The significance of learners' errors. International Review of Applied Linguistics 9: 147-159.

CORDER, S. P. (1981) Error Analysis and Interlanguage. Oxford: Oxford University Press.

D'ANNUNZIO, B./G. SERAGIOTTO (2007) La valutazione e l'analisi dell'errore. http://venus.unive.it/filmi

ELLIS, R. (1994) Learners errors and error analysis. R. Ellis (a cura di), The study of second language acquisition (first edition). Oxford: Oxford University Press, 47-72.

GASS, S. M./L. SELINKER (2008) Second language acquisition: An introductory course (third edition). New York: Routledge.

HARTSHORN, K. J./N. E. EVANS (2015) The Effects of Dynamic Written Corrective Feedback: A 30-Week Study. Journal of Response to Writing 1/2, 6-34.

KRASHEN, S. D. (1982) Principles and practice in second language acquisition. New York: Prentice Hall.

L'enciclopedia dell'italiano Treccani 10. avgust $2017 \mathrm{http}: / /$ www.treccani.it

LENNON, P. (1991) Error: Some Problems of Definition, Identification and Distinction. Applied Linguistics 12, 180-196.

LARSEN-FREEMAN, D. (2001) Teaching grammar. M. Celce-Murcia (a cura di), Teaching English as a second or foreign language (third edition). Boston: Heinle \& Heinle, 251-266.

LAH, M. (2006) Pisna kompetenca pri tujem jeziku: primer slovenskih dijakov francoščine. Vestnik 40/1-2, 23 - 34

LONG, M. H. (2003) Stabilization and fossilization in interlanguage development. C. J. Doughty/M. H. Long (a cura di), Handbook of second language acquisition. Oxford: Blackwell, 487-535.

Quadro comune europeo di riferimento per le lingue: apprendimento, insegnamento, valutazione (2002) Milano: La Nuova Italia-Oxford.

MERTELJ, D. (2003) Naloge za urjenje slovničnih struktur v učbenikih italijanščine kot tujega jezika. Vestnik 37/1-2, 131-147. 
MERTELJ, D. (2005) Poučevanje nekaterih podrednih skladenjskih vzorcev pri predmetu »italijanščine « kot tuji jezik v slovenskih gimnazijah: sedanje stanje in predlogi za spremembe. Doktorska disertacija. Ljubljana: Univerza v Ljubljani, Filozofska fakulteta

MEZZADRI, M. (2015) La correzione degli errori. M. Mezzadri (a cura di), I nuovi ferri del mestiere: manuale di glottodidattica. Torino, Loescher-Bonacci, 269-282.

MIKLIČ, T. (1992) Tendenze nella scelta delle forme verbali in testi prodotti da apprendenti sloveni: in cerca di spie dell'interferenza. B. Moretti/D. Petrini/S. Bianconi (a cura di), Linee di tendenza dell'italiano contemporaneo - Atti del XXV congresso della Società di linguistica italiana. Roma: Bulzoni, 475-492.

MIKLIČ, T./M. OŽBOT (2007) Teaching the uses of Italian verb forms to Slovene speakers. Linguistica 47, 65-76.

PREMRL, M. (2010) Commento delle scelte delle forme verbali nei cloze degli studenti di italianistica e di traduzione (identificazione dei punti critici e confronto tra i gruppi). Linguistica, 49/1, 155-197.

PREMRL, M. (2016) Izražanje določnosti v italijanščini in slovenščini: doktorska disertacija. Ljubljana: Univerza v Ljubljani, Filozofska fakulteta.

PAWLAK, M. (2014) Error Correction in the Foreign Language Classroom: Reconsidering the Issues. Heidelberg: Springer.

REYMÓNDEZ FERNÁNDEZ, I./M. G. SANTIAGO ALONSO (2004) Gramática mínima de errores. Análisis de errores del español producidos por aprendices eslovenos. Ljubljana: G. M. Santiago Alonso.

RUSSELL, V. (2009) Corrective feedback, over a decade of research since Lyster and Ranta (1997): Where do we stand today? Electronic Journal of Foreign Language Teaching 6: 21-31.

SAVILLE-TROIKE, M. (2006) The linguistics of Second Language Acquisition. M. Saville-Troike (a cura di) Introducing Second Language Acquisition. New York: Cambridge University Press, 31-65.

SELINKER, L. (1972) Interlanguage. IRAL; International Review of Applied Linguistics in Language Teaching 10/3, 209-231.

SERIANNI, L. (2006) Prima lezione di grammatica. Bari: Editori Laterza.

SERIANNI, L. (2010) Grammatica italiana. Torino: UTET.

SOSNOWSKI, R. (2010) Errori dovuti a interferenze negli scritti degli apprendenti polacchi (livello intermedio avanzato). Ricerca corpus based. Romanica Cracoviensia 10, 135-156.

TAYLOR, G. (1986) Errors and explanations. Applied Linguistics 7/2, 144-166.

TRENKIĆ, D. (2009) Accounting for patterns of article omissions and substitutions in second language production. R. Hawkins e M. García Mayo (a cura di) Second language acquisition of articles: Empirical findings and theoretical implications. Amsterdam: John Benjamins, 115-143. 
ŽELE, A. (2009) O povedkovniku oziroma povedkovniškosti. Jezikoslovni zapiski 15/12, 63-72.

ŽUGELJ, N. (2005) Analiza napak slovenskih študentov v francoskem pisnem diskurzu: magistrska naloga. Ljubljana: Univerza v Ljubljani, Filozofska fakulteta.

\section{POVZETEK}

\section{Analiza napak pri pisnem sporočanju študentov italijanščine}

V prispevku so najprej predstavljena nekatera teoretična izhodišča: zgodovinski pregled obravnavanja napake v učenju/poučevanju tujega jezika, vrste napak, kriteriji na osnovi katerih določamo napake ter vzroki za njihov nastanek. V nadaljevanju sta predstavljena korpus pisnih izdelkov študentov italijanščine ter podrobna analiza najpogostejših napak s primeri. Avtorica meni, da so za doseganje visoke ravni jezikovne kompetence nujni učenčevo zavedanje lastnih napak in razmislek o napakah na eni strani ter učiteljevo poznavanje tipičnih napak govorcev slovenščine (v našem primeru študentov italijanščine) pri učenju italijanskega jezika kot tujega jezika. Vzroke, ki vplivajo na napake in jih je bilo mogoče določiti, smo lahko razdelili v tri skupine: interference maternega jezika, interference drugih tujih jezikov, ki jih študirajo ali govorijo študenti ter značilnosti samega jezika, ki se ga učijo. Pri posameznih napakah so vzroki, v kolikor jih je bilo mogoče določiti ali nanje sklepati, tudi podrobneje pojasnjeni. Kljub relativno visokemu številu analiziranih izdelkov se je korpus izkazal za neprimernega in premajhnega za analizo nekaterih napak, predvsem tistih, ki izhajajo iz rabe glagolskih oblik.

Ključne besede: napake, analiza napak, italijanščina, pisno sporočanje

\section{ABSTRACT}

\section{Analysis of Errors in Written Production of Students of Italian as a Foreign Language in the University Context}

The paper begins with a presentation of a theoretical framework and also includes an overview of the treatment of learners errors within different approaches of Second Language Acquisition. Also, the types of errors, the criteria for their identification and, finally, their causes, are discussed. The second part deals with a corpus consisting of the learners' written products, in which the most frequent errors are described and explained (the learners are students of the BA course in Italian at the Faculty of Arts of Ljubljana). The author believes that in order to achieve high levels of competence in foreign language, it is necessary for the students to become aware of their errors and to reflect on them with the help of the teacher, who should be familiar with the types of errors the 
students are like to make. The purpose of the analysis was to find out the most frequent errors and to identify and describe their causes. Although it was not possible to determine the causes of all the students' errors, three main sources of the most common errors were identified: a. the students' L1, b. the characteristics of the language which is being learned, c. other foreign languages the student is learning or already knows. Although the corpus consists of a relatively high number of written assignments, it appeared too small to allow a reliable analysis of some types of errors to be carried out, particularly those related to the use of verb forms.

Key words: errors, error analysis, Italian, written production

\section{RIASSUNTO}

\section{Analisi degli errori nella produzione scritta degli studenti di italiano come ls a livello universitario}

All'inizio del contributo vengono esposte le premesse teoriche: la storia della concezione dell'errore nei diversi metodi dell'insegnamento della lingua straniera, i tipi di errori, i criteri i base ai quali è possibile identificare un errore e le cause degli errori. Di seguito sono presentati il corpus dei compiti scritti degli studenti e l'analisi degli errori più tipici e frequenti degli studenti slovenofoni con esempi. L'autrice è del parere che per raggiungere alti livelli di competenza in una lingua straniera sono indispensabili la consapevolezza dei propri errori e la riflessione linguistica su di essi da parte dell'apprendente, guidata da un insegnante che conosca gli errori tipici dei propri studenti (nel nostro caso si tratta di studenti slovenofoni del corso di Laurea Triennale in Lingua e letteratura italiana della Facoltà di lettere e Filosofia di Lubiana). Proprio per questa ragione il contributo si propone di descrivere gli errori più frequenti e di stabilire e descrivere le loro cause. Benché non sia stato possibile risalire a tutte le cause degli errori, l'analisi individua e conferma le tre principali fonti degli errori: la L1, altre lingue straniere che gli studenti studiano o conoscono e la lingua da apprendere. Nonostante il corpus fosse composto da un numero relativamente alto di compiti scritti, si è dimostrato troppo limitato per l'analisi di alcuni errori, specialmente per l'analisi degli errori nell'uso delle forme verbali.

Parole chiave: errori, analisi di errori, italiano, produzione scritta 